\title{
Developing disease
}

\author{
When pathways vital for development go awry, the consequences can be disastrous. This collection of reviews \\ highlights emerging translational aspects of developmental biology at a time when the first clinical applications \\ are taking shape.
}

Understanding the molecular mechanisms that drive cellular processes during animal development has been the aim of developmental biology research for many years; yet the field has only recently whole heartedly embraced molecular and cell-biological approaches that complement its traditional repertoire of genetic and embryology techniques. As a result, modern developmental biology now finds itself at the interface between many other disciplines, as new biochemical, imaging, genomic and systems approaches are developed and implemented. As we learn more about the molecular mechanisms controlling development, the traditional schism between cell and developmental biology is set to disappear.

Throughout development, morphogenetic processes drive the formation of highly specialized organs. Once development is completed, homeostasis takes over; notably, the repair, and maintenance, of adult tissues such as bone, muscle, liver and skin also uses developmental signalling pathways and stem cells. It is perhaps not surprising that ectopic activation of the signalling pathways that control normal development and homeostasis can lead to hyperproliferative conditions, resulting in cancers. Moreover, morphogenetic pathways that are vital for development are hijacked by invasive cancer cells during metastasis. A plethora of other key diseases such as diabetes are also rooted in defective developmental processes. As a result, many of the findings made originally in a purely developmental context are now becoming significant in clinically relevant research. It is thus an excellent time to highlight this transitional aspect of basic cell biology.

It would be futile to try to cover the whole range of developmental pathways that have links to human disease in a single issue. Rather, we have chosen to highlight a few research areas that illustrate the diverse manner in which developmental pathways go awry and cause disease. It would be equally difficult to discuss all the model organisms used to study these processes, and so Nadia Rosenthal and Steve Brown focus on perhaps the most clinically relevant model, the mouse, and discuss its importance for studying human diseases.

During development, cell polarity directs the formation of tissue structures and specifies cell fates through the asymmetric distribution of determinants. Loss of cell polarity is commonly observed in advanced tumours, and Andreas Wodarz and Inke Näthke present the growing evidence from studies of Drosophila and mammals that loss of cell polarity and cell-cell adhesion can initiate tumorigenesis. Jeremy Green and Lance Davidson investigate how polarity participates in Xenopus convergent extension - tissue elongation through cell rearrangements. They suggest that malfunctioning of this process during organogenesis in late development causes cranio-facial defects in humans.

As development progresses, multipotent stem cells commit to specific lineages. Resolving the molecular mechanisms that drive this is a long-standing goal for developmental biologists. The Hedgehog morphogen controls the specification of most tissues and organs, and, although the exact molecular mechanisms underlying the initial steps of the signalling cascade are still unclear, mutations in genes controlling this pathway have been linked to both hereditary and sporadic forms of cancer. Rajat Rohatgi and Mathew Scott highlight how trafficking of Hedgehog-signalling components influences the outcome of the morphogen response and how therapeutic action on these trafficking steps could be used to treat these cancers. Although commitment to a specific fate is essential during development, several signalling pathways are able to re-direct differentiation towards an alternative fate. Although this plasticity is required for normal development, it can give rise to a variety of diseases if triggered in the adult, as illustrated by Rik Derynck and Rosemary Akhurst for TGF- $\beta$ signalling.

One of the long-term goals for researchers studying stem cells is to generate stem-cell-based therapies. The road to this goal passes through an ethical minefield. Robin Lovell-Badge and Justin St John comment on the ethics and science of developing human-animal cytoplasmic hybrid (cybrid) embryos - obtained by injecting human nuclear material into animal oocytes - with the purpose of generating human embryonic stem cells.

We hope that these articles illuminate and celebrate both the basic interest in understanding the mechanisms that drive development and the remarkable clinical potential of this knowledge. To illustrate the fading of the frontiers between clinicians and developmental biologists as we learn more about how derailed developmental pathways cause disease, the symbol of medicine (a snake whose shedding skin symbolizes re-birth, entwined with a staff that represents authority and is historically associated with Asclepius, the Greek god of medicine) is depicted on the cover of the Focus merged with examples of the model organisms that form the pillars of developmental biology.

We are pleased to acknowledge the financial support of the March of Dimes foundation, as well as the Institute of Biochemistry and Cell Biology, Shanghai, and the Guangzhou Institute of Biomedicine and Health, both of the Chinese Academy of Sciences, for this focus. As always, Nature Cell Biology carries sole responsibility for all editorial content. The articles are freely available for six months and canbeaccessed throughadedicated website (http://www.nature.com/ ncb/webfocus/developmentdisease), which also includes a collection of recent papers highlighting the links between developmental biology and human-disease pathologies from Nature family journals and $E M B O J$. 